\title{
A Novel Strategy for Improving the Counter Propagation Artificial Neural Networks in Classification Tasks
}

\author{
Sara Belattar, Otman Abdoun, and El Khatir Haimoudi
}

\begin{abstract}
Counter-Propagation-Artificial-Neural-Networks (C P-ANNs) have been applied in several domains due to their learning and classification abilities. Regardless of their strength, the CP-ANNs still have some limitations in pattern recognition tasks when they encounter ambiguities during the learning process, which leads to the inaccurate classification of the Kohonen-Self-Organizing-Map (K-SOM). This problem has an impact on the performance of the CP-ANNs. Therefore, this paper proposes a novel strategy to improve the CP-ANNs by the Gram-Schmidt algorithm (GSHM) as a pre-processing step of the original data without changing their architecture. Three datasets examples from various domains, such as correlation, crop, and fertilizer, were employed for experimental validation. To obtain the results, we relied on two simulations. The first simulation uses CP-ANNs, and the datasets are inputted into the network without any prior pre-processing. The second simulation uses MCPANNs, and the datasets are pre-processed through the GSHM block. Experiment results show that the proposed MCP-ANNs recognize all patterns with a classification accuracy of $100 \%$ versus $62.5 \%$ for CP-ANNs in the Correlation Dataset. Furthermore, the proposed MCP-ANNs reduce the execution time and training parameter values in all datasets versus $C P$. ANNs. Thus, the proposed approach based on the GSHM algorithm significantly improves the performance of the $\mathrm{CP}$ ANNs.
\end{abstract}

Index Terms-Counter Propagation Artificial Neural Networks (CP-ANNs), Classification performance, Data Preprocessing, Gram-Schmidt Algorithm (GSHM), Kohonen- SelfOrganizing-Map (K-SOM), Modified Counter Propagation Artificial Neural Networks (MCP-ANNs).

\section{INTRODUCTION}

A rtificial Neural Network (ANN) is a distinguished and acknowledgeable field in solving complex problems which are challenging tasks in various issues such as classification, description, prediction, diagnostic, and pattern recognition (PR). The power of the ANN is due to its

Manuscript received July 20, 2021; revised November 15, 2021. Date of publication January 24, 2022. Date of current version January 24, 2022. The associate editor prof. Matko Šarić has been coordinating the review of this manuscript and approved it for publication.

Authors are with the Abdelmalek Essaadi University, Polydisciplinary Faculty, Computer Science Department, Advanced Science and Technologies Laboratory, Larache, Morocco (e-mails: sarah.belattar12@gmail.com, abdoun.otman@gmail.com, helkhatir@gmail.com).

Digital Object Identifier (DOI): 10.24138/jcomss-2021-0121 inspiration from the biological human nervous system that includes intelligent features like self-adapting, learning ability, and generalization of the results. Furthermore, the ANN is among the classification algorithms used in several classification tasks. In the ANN, the classification accuracy depends on pattern recognition performance.

The ANN has been incorporated into numerous areas, including medical data classification [1-3], agriculture prediction [4-6], diagnostic industry [7-9], biometric [10], [11], and face recognition [12]. The performance of the ANN in the previously cited works showed significant results and is considered an effective tool in solving various complex problems. These achievements can be attributed to the effective use of the ANN in PR because this latter has a high weight in the development of research and offers a solution to a variety of challenges. Nevertheless, an improvement step of the ANN is required to avoid some issues faced in pattern recognition systems, which may have a negative impact on the ANN classification performance. Since PR classifies the patterns in a specific class, sometimes the classification results may differ from the results assumed through the lack of recognition of some patterns (unknown class), resulting in a bad decision. For this purpose, many works suggested new strategies for improving the ANN in terms of classification [13-15]. These strategies achieved higher classification accuracy with minimum errors than original neural networks.

Several models of the ANN have been vastly used in pattern recognition, such as Radial Basis Function Network (RBFN), Multi-layer Perceptron (MLP), Counter Propagation Artificial Neural Networks (CP-ANNs), Kohonen Self-Organizing-Map (K-SOM), Convolutional Neural Network (CNN), and so on). In this study, we suggested the CP-ANNs. The purpose of choosing this model is its capability to combine both unsupervised and supervised learning. In Addition, the CPANNs are a popular network [16] and are extensively used in numerous areas [17-19] through their abilities to solve the issues of classification, clustering, and recognition tasks. Moreover, pattern recognition comprises two learning modes (Unsupervised and supervised) [20]. Generally, the CP-ANNs are classified as a supervised learning network with two layers: the K-SOM and the Grossberg. The objective of the first layer is to regroup the data into the cluster by making a correct classification of all patterns and the second layer (Grossberg) depends on the classification performance of the 
first layer (K-SOM) to reach some expectations. For the data that suffer linear regularities between their patterns, The KSOM may fail to increase the number of correct classifications due to the low rate of their patterns recognition. Hence, the Grossberg layer gives incorrect outputs. As a result, the classification accuracy decreases and it is regarded as one of the major inconveniences of the CP-ANNs in all tasks. Therefore, improvement is necessary within the supervised learning. In order to overcome this limitation, there are different approaches to detect and remove the drawbacks encountered during the learning process. For that reason, we must search the appropriate strategy to eliminate them, and this represented a challenging task. Thus, this paper proposes a new strategy for improving the CP-ANNs by adding a preprocessing step of the Gram-Schmidt algorithm. The MCPANNs use the GSHM algorithm to remove all the ambiguities and drawbacks detected from data that would improve the rate of pattern recognition and classification accuracy of the classical CP-ANNs. The GSHM algorithm is a linearly orthogonalized algorithm that constructs the $(\mathrm{K}, \mathrm{R})$ components from the given ( $\mathrm{r}$ x c) matrix. This algorithm can enhance the level of the K-SOM pattern recognition. As a consequence, the classification of the CP-ANNs network becomes more accurate.

In this work, three datasets from various domains such as correlation, crop, and fertilizer datasets were used for training and testing the proposed MCP-ANNs approach. The main goal of choosing these datasets is to prove the performance of the proposed MCP-ANNs in data with relation complexes and in data that doesn't have any problems (see Fig. 1). Hence, the effectiveness of the proposed approach will be compared to the CP-ANNs effectiveness.

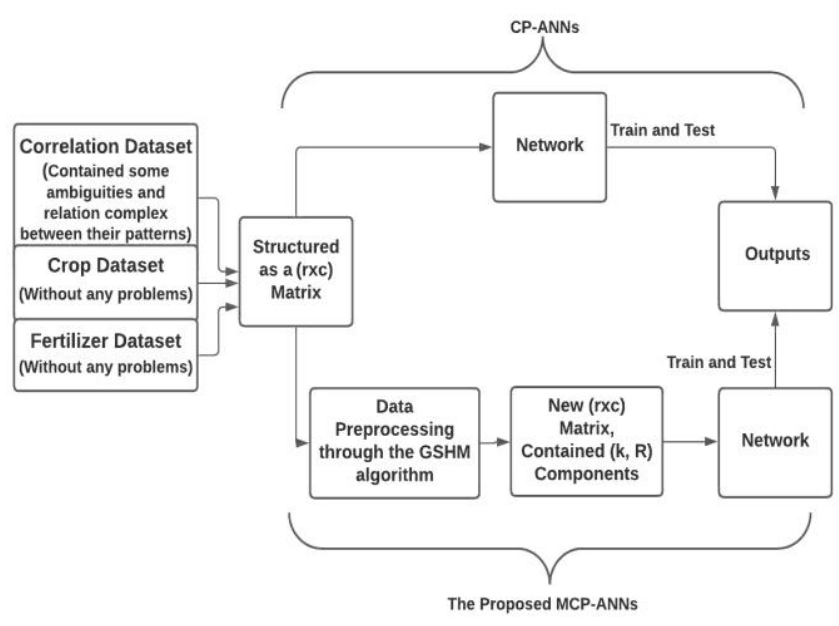

Fig. 1. Process of the dataset implementation used in the CP-ANNs and the proposed MCP-ANNs.

The main contributions of this study are summarized as follows:

- This study contributes by proposing a novel strategy based on a combination of the CP-ANNs and the Gram-Schmidt algorithm (GSHM).

- The GSHM algorithm is employed to increase the rate of pattern recognition by removing all ambiguities detected, which improves the classification accuracy in the supervised learning during the learning process.

- Our proposed MCP-ANNs approach achieves a better classification with high accuracy in the correlation dataset.

- In terms of the $\mathrm{ANN}$, the long training represents a second challenge issue after the classification problem. For that, the proposed MCP-ANNs reduce the execution time in all datasets used. Besides that, the MCP-ANNs minimize some (or all) training parameter values compared to the CP-ANNs.

Furthermore, our paper can provide additional contributions in comparison with previous works on this subject, which are illustrated as follows:

- According to this publication [21], the proposed GSHM algorithm outperforms the PCA in the execution time term since the GSHM algorithm is linear and the PCA algorithm is iterative. Thus, the GSHM is faster than PCA in data pre-processing. Additionally, The GSHM gives more precise values than PCA.

- Based on this publication [15], the linear model based on the Kalman filter improved the classification accuracy of the Neural Network by $94,93 \%$ in the Iris dataset (the example of PR dataset) compared to the proposed MCP-ANNs, which reach an accuracy of $100 \%$ in the Correlation dataset (our PR dataset example).

- The authors of this study [22] examined each ANN model in PR, and they obtained the accuracy between 70.11 and $89.97 \%$. Thus, the proposed MCP-ANNs can be added to [22] ANN models by the accuracy of $100 \%$ in pattern recognition.

The remainder of the article is structured as follows. Section II gives a few strategies employed for improving the classification accuracy of the Neural Networks. Section III describes the datasets used for experimental validation. Section IV explains the CP-ANNs and the proposed MCPANNs. Section V presents and compares the performance results achieved by the CP-ANNs and the proposed MCPANNs. Section VI discusses the empirical results. And finally, the conclusion and perspective are determined by Section VII.

\section{RELATED WORK}

The Principal Component Analysis (PCA) method was proposed in this work [21] to improve the classification accuracy of the K-SOM networks by eliminating the drawbacks encountered during the learning process. In this study, their proposed strategy removed the relation complexes between patterns, resulting in better classification performance when compared to the standard K-SOM. The Gram-Schmidt algorithm was suggested in our first article [23] to increase the classification accuracy of the K-SOM in unsupervised 
learning. For validation in this study, we used two datasets. The experimental results showed that our proposed approach outperforms the classical K-SOM by removing all ambiguities detected in data that suffer some problems between their patterns. Therefore, the modified K-SOM produced a classification accuracy of $100 \%$ by reducing the execution time and the training parameter values versus the K-SOM. The combination of both Learning Vector Quantization and SelfOrganizing Map was employed [24], which was named Weighted-Self Organizing Map (W-SOM). The main objective of their study is to enhance the prediction accuracy of crops and weather for the Mysore region of India. The experimental results demonstrated that their proposed $\mathrm{W}-\mathrm{SOM}$ improved the accuracy versus the existing methods such as SOM, KNN, and ENN. The linear model based on the Kalman filter was developed to improve the classification performance of the Neural Networks (NN) [15]. Their proposed technique used the Kalman filter to evaluate the linear model parameters, which is considered post-processing of the original Neural Network. For validation, they used five datasets from various domains with different characteristics. Their results demonstrated high classification accuracy than NN. Additionally, their proposed method reduced the error of NN.

\section{DATASET INTRODUCTION}

To evaluate the effectiveness of the proposed MCP-ANNs approach, three examples of the dataset were selected from various domains and with different natures (see Tables I, II, and III). This evaluation aims to study some limitations of the CP-ANNs and improve them. The concise descriptions of each dataset used are portrayed below.

1. The correlation dataset represents our example of a pattern recognition dataset. This latter is one of the datasets that may be encountered during the learning process, and it includes some ambiguities [21]. The correlation dataset consists of two similar input vectors ( 1 and 5), four input vectors that have linear regularity and dependence between its object components $(0,4,2$, and 6), and finally, two normal input vectors (3 and 7). For desired outputs, we gave them a different number code. And for similar vectors, we gave them the same number code (see Table I).

2. The crop dataset is used to recommend a suitable crop based on specific parameters related to soil and atmosphere, and it was collected from India [25]. This dataset contains seven parameters: $\mathrm{Ph}, \mathrm{N}, \mathrm{P}, \mathrm{K}$, Depth, Temperature, and Rainfall (see Table II). We randomly divided the crop dataset (11 patterns) into training sets $(85 \%)$, and the remaining $15 \%$ was used for testing and validating the models.

3. The fertilizer dataset is employed to recommend the appropriate fertilizer type according to various parameters such as temperature, humidity, moisture, soil type, crop type, nitrogen, potassium, and phosphorus [26] (see Table III). We randomly split the fertilizer dataset (40 patterns) into training sets (95\%), testing and validating sets $(5 \%)$.
Since the CP-ANNs only take numerical values as input and output in the training phase, we offered a number code for each desired output and each parameter related to crop and soil types. The number codes were chosen randomly (see Table V).

TABLE I

THE CORRELATION DATASET

\begin{tabular}{|l|l|l|}
\hline Input Vector $\mathbf{N}^{\mathbf{0}}$ & Learning Data & Desired Outputs \\
\hline 0 & $8,4,6$ & 1 \\
\hline 1 & $5,6,7$ & 2 \\
\hline 2 & $3,5,4$ & 3 \\
\hline 3 & $11,27,39$ & 4 \\
\hline 4 & $4,2,3$ & 5 \\
\hline 5 & $5,6,7$ & 2 \\
\hline 6 & $9,15,12$ & 6 \\
\hline 7 & $13,35,42$ & 7 \\
\hline
\end{tabular}

TABLE II

THE CROP DATASET

\begin{tabular}{|l|l|l|l|l|l|l|l|}
\hline \multicolumn{7}{|l|}{$\begin{array}{l}\text { The parameters according to atmosphere and soil } \\
\text { (Learning Data) }\end{array}$} & $\begin{array}{l}\text { Crop Type } \\
\text { (Desired } \\
\text { Outputs) }\end{array}$ \\
\hline PH & N & P & K & Dep & Temp & Rain & Cotton \\
\hline 8.5 & 100 & 50 & 50 & 30 & 33 & 1200 & Suarcane \\
\hline 7 & 175 & 100 & 100 & 60 & 30 & 750 & Sugaren \\
\hline 8 & 80 & 40 & 40 & 50 & 30 & 1000 & Jowar \\
\hline 7 & 40 & 20 & 25 & 15 & 28 & 600 & Bajra \\
\hline 7 & 30 & 75 & 15 & 19 & 27 & 800 & Soybeans \\
\hline 8 & 100 & 25 & 0 & 40 & 20 & 500 & Corn \\
\hline 7 & 100 & 50 & 50 & 15 & 22 & 90 & Rice \\
\hline 8.5 & 100 & 50 & 50 & 30 & 24 & 1400 & Wheat \\
\hline 6 & 25 & 50 & 30 & 20 & 24 & 1250 & Groundnut \\
\hline
\end{tabular}

TABLE III

THE FERTILIZER DATASET

\begin{tabular}{|c|c|c|c|c|c|c|c|c|}
\hline \multicolumn{8}{|c|}{$\begin{array}{l}\text { The specific parameters for recommending the suitable } \\
\text { fertilizer (Learning Data) }\end{array}$} & \multirow{2}{*}{$\begin{array}{l}\text { Fertilizer } \\
\text { Name } \\
\text { (Desired } \\
\text { Outputs) }\end{array}$} \\
\hline Temp & $\mathrm{H}$ & $\mathrm{M}$ & $\begin{array}{l}\text { Soil } \\
\text { Type }\end{array}$ & $\begin{array}{l}\text { Crop } \\
\text { Type }\end{array}$ & $\mathrm{N}$ & Pot & Pho & \\
\hline 26 & 52 & 38 & Sandy & Maize & 37 & 0 & 0 & Urea \\
\hline 29 & 52 & 45 & Loamy & $\begin{array}{l}\text { Sugar- } \\
\text { cane }\end{array}$ & 12 & 0 & 36 & DAP \\
\hline 28 & 54 & 46 & Clayey & Paddy & 35 & 0 & 0 & Urea \\
\hline 33 & 64 & 50 & Loamy & Wheat & 41 & 0 & 0 & Urea \\
\hline 27 & 54 & 28 & Clayey & Pulses & 13 & 0 & 40 & DAP \\
\hline 25 & 50 & 65 & Loamy & Cotton & 36 & 0 & 0 & Urea \\
\hline 26 & 52 & 31 & Red & $\begin{array}{l}\text { Grou- } \\
\text { ndnuts }\end{array}$ & 14 & 0 & 41 & DAP \\
\hline 6 & 60 & 43 & Son & Mill & 15 & 0 & 41 & $\mathrm{D}$ \\
\hline
\end{tabular}

\section{THE PRoposed ANN BASED SysteMS}

\section{A. Artificial Neural Networks}

The ANNs are based on a biological neural network that can learn, adapt, and generalize results more effectively than other methods [27]. These proprieties allow solving complex problems in various systems [28-30]. In addition, ANNs have been widely employed in nonlinear systems, and they are dominant in numerous application areas [31], [32]. Although to these mentioned capabilities of the ANNs, sometimes they may fail to achieve high classification accuracy due to the lack of pattern recognition. This problem occurs from some detected drawbacks during the learning process. For that reason, we propose a new strategy to improve the ANNs in their performance. The proposed approach makes the ANNs more robust. There are several ANN models; in this study, we 
suggest the CP-ANNs since they are frequently utilized in pattern classification tasks.

\section{B. Counter Propagation Artificial Neural Networks}

The Counter-Propagation Artificial Neural Networks are a popular model of artificial neural networks developed by Hecht Nielsen (1987) [33], [34]. This model serves as a fast network in data classification with high accuracy. The main reason for the CP-ANNs success is their capabilities to solve several problems in clustering and classification tasks [35-37], allowing us to build effective intelligent systems. The CPANNs connect the Kohonen-Self-Organizing Map and Grossberg successively. For that reason, the CP-ANNs use two modes of learning; unsupervised and supervised learning. However, the CP-ANNs represent the supervised network model. The architecture of the CP-ANNs is composed of three-layer (i.e., input layer, Kohonen layer, and Grossberg Layer). The input layer aims to distribute the data to the learning processes and is represented by vector $X$. The second layer is named the Kohonen Self-Organizing Map (K-SOM) and is among the Artificial Neural Network models. Moreover, the K-SOM is the best-known and commonly used in data classification and clustering [38], [39].

In the CP-ANNs, the K-SOM was trained in an unsupervised manner and is based on the values given by the input layer to determine the winning nodes (or neurons), which are the observation situations that we have. The winning neurons are presented by 1 and 0 for all other neighboring neurons (accreditation regime), and for calculating it, there are many methods, but the most used methods are the Euclidean Distance and the Product Scalar. The principle of these techniques is to calculate the distance between all input vectors $X$ and weight vectors $W$ of each neuron, and the shortest distance represent the winning neuron. The outputs values of the K-SOM are the inputs values of the third layer (Named Grossberg). The objective of the Grossberg layer is to achieve the desired outputs that have been classified correctly in the Kohonen layer. Additionally, the Grossberg layer was trained in a supervised mode manner. The CP-ANNs outputs are represented by vector $Y$. Thus, by linking the Kohonen and the Grossberg layers, we attain the architecture of the CP-ANNs (see Fig. 2).

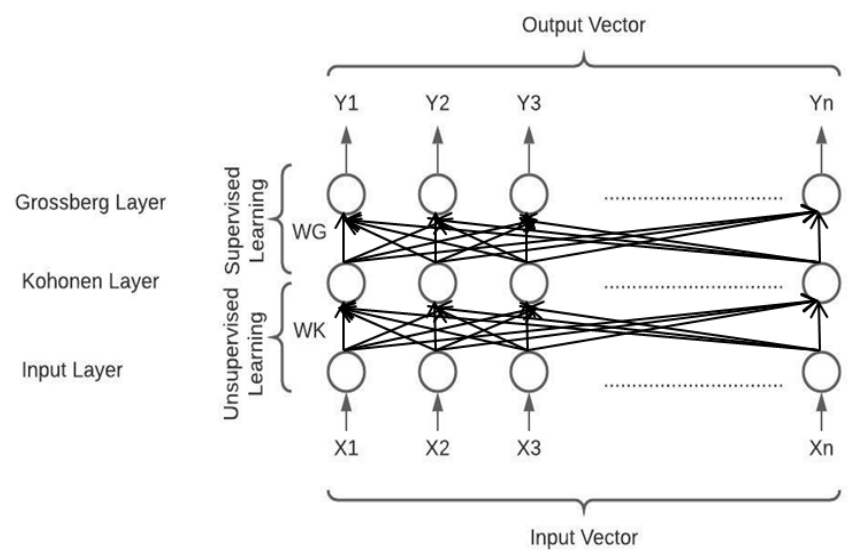

Fig. 2. The architecture of the CP-ANNs.
As can be seen from Fig. 2, the learning Procedure of the Classical Counter-Propagation Artificial Neural Networks begins with the Kohonen Self-organizing map. Thus, we must create the map of the K-SOM that contains the required number of neurons and weights in order to connect the input data with all neurons in the map. The initial weights are initialized randomly between zero and one. For training the patterns, the input data is formed as a ( $\mathrm{r}$ x c) matrix and must be normalized to speed up the learning time and improve the convergence of the neural network. The equation of the normalization step [40] can be written as follows.

$$
X_{\mathrm{i}}=X_{\mathrm{i}} / \sqrt{\sum_{j=0}^{n-1} X_{\mathrm{j}}^{2}}
$$

where $X_{i}$ : is the input vector of $\mathrm{i}^{\text {th }}$ element.

In the next step, the Euclidean Distance (ED) metric is used in this algorithm to determine the winning neuron by calculating the distance between the input vector $X$ and the weight vector $W K_{j}$ (of each neuron). The nearest distance between the input vector and weight vector is the winning neuron $j$ and is given by the following equation [21].

$$
j:\left\|W K_{j}-X\right\| \leq\left\|W K_{o}-X\right\| \quad \forall_{o}
$$

After determining the winning neuron by the Euclidean Distance metric, the weights of the winning neuron and all its neighbors must be corrected and updated using the following equation [23], [41-43].

$$
W K_{i j}(t+1)=W K_{i j}(t)+L(t) f(t) \cdot\left\lfloor y_{\mathrm{i}}-W K_{i j}(t)\right\rfloor
$$

where $W K_{i j}(t+1)$ is the updated synaptic weight at iteration $\mathrm{t}+1, W K_{i j}(t)$ is the synaptic weight before updating at iteration $\mathrm{t}, L(t)$ is the learning rate at iteration $\mathrm{t}, f(t)$ is the neighborhood function. $y_{\mathrm{i}}$ is the value of the $(i)$ winning neuron.

In order to complete the Counter Propagation Artificial Neural Networks, we have to create the third layer (Grossberg). Therefore, the values obtained at the output of the K-SOM are transmitted to the input of the Grossberg layer. The equation that calculates the network outputs is as follows.

$$
y_{k}=\sum_{i}^{N} u_{\mathrm{i}} \cdot W_{i k}
$$

where $y_{k}$ : is the output of the network. $u_{i}$ is the $i^{\text {th }}$ output of the K-SOM. And $W_{i k}$ is the synaptic weight within $i^{\text {th }}$ neuron of the K-SOM layer and $k^{\text {th }}$ neuron of the Grossberg layer.

In the end, the synaptic weight of the Grossberg layer must be updated using the formula below to reach some expectations.

$$
W G_{i j}(t+1)=W G_{i j}(t)+\beta \cdot\left(y_{d}-W G_{i j}(t)\right) \cdot u_{i}
$$

where $\beta$ : is the learning rate, and $y_{d}$ is the desired output. 


\section{Modified Counter Propagation Artificial Neural Networks}

The ultimate goal of the supervised CP-ANNs is to train the network to arrive at some expectations. These expectations must be very close to desired outputs. However, when the $\mathrm{CP}$ ANNs encounter drawbacks during the learning, the network is unable to achieve better performance due to its failure in recognizing some patterns by the K-SOM layer, resulting in inaccurate classification. Therefore, to increase the CP-ANNs classification accuracy, the Gram-Schmidt algorithm that generates the new data by removing all drawbacks detected can be considered as a pre-processing step. In the linear algebra, the Gram-Schmidt mathematical algorithm is a technique of orthogonalization to generate the new set of orthogonal vectors in Euclidean space from the original matrix (or original data) [44], [45]. Consequently, we can conclude that the GSHM algorithm is a new technique for pattern recognition, among other algorithms like PCA. By combining the GSHM algorithm and the CP-ANNs, we achieve the MCPANNs (see Fig. 3).

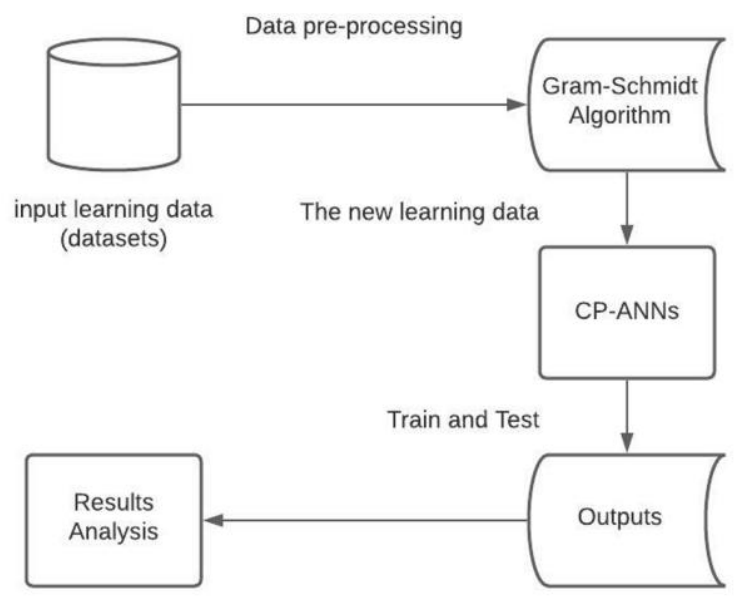

Fig. 3. Flowchart design of the proposed MCP-ANNs approach.

In this work, we use the modified Gram-Schmidt algorithm. To develop the GSHM algorithm, we have to apply all the following steps. We refer to [23].

1. Firstly, the dataset (or learning data) must be organized into $\mathrm{a}(\mathrm{r} \times \mathrm{x}$ ) matrix in order to facilitate their processing by the GSHM algorithm.

2. Secondly, each matrix row will be represented by a point in 3-dimensional space (see Fig. 4), where the point $(1,2$, and $\mathrm{m})$ in 3-dimensional space corresponds to the $1 \mathrm{st}, 2 \mathrm{nd}$, and $\mathrm{m}^{\text {th }}$ row of the matrix.

3. Each row of the matrix has $(K, R)$ components. Therefore, to calculate these components of the new matrix generated by the GSHM algorithm, we start choosing the point farthest from the initial coordinates. After that, we draw a vector that starts with the chosen point $(l)$ until the initial coordinates and perpendicular to it, passing through the origin of the coordinate. We draw a plan with the following equation.

$$
a_{l 1} a_{1}+a_{l 2} a_{2}+a_{l 3} a_{3}=0
$$

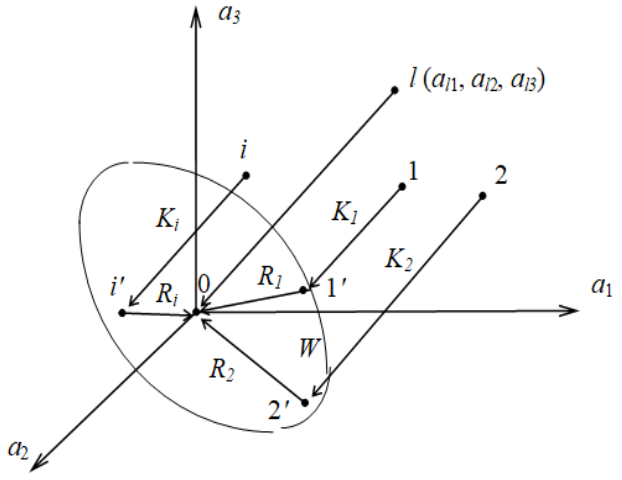

Fig. 4. 3-dimensional space.

4. In a given space, the distances of an arbitrary point regarding the drawn plan are equal to the absolute value of (Dist) (see formula below).

$$
\text { Dist }=\frac{a_{l 1} a_{l}+a_{l 2} a_{2}+\ldots+a_{l 3} a_{3}}{\sqrt{a_{l 1}^{2}+a_{l 2}^{2}+\ldots+a_{l 3}^{2}}}
$$

5. The value of the resultant expression for (Dist) corresponds to the value of the $K_{l}$ coefficient calculated by the following function.

$$
K_{l}^{(\mathrm{j})}=\sum_{\mathrm{b}=1}^{\mathrm{m}}\left(M_{l, \mathrm{~b}}^{(\mathrm{j})} \cdot M_{c, \mathrm{~b}}^{(\mathrm{j})}\right) / \sum_{\mathrm{b}=1}^{\mathrm{m}}\left(M_{c, \mathrm{~b}}^{(\mathrm{j})}\right)^{2}
$$

6. $\mid$ Dist $\mid$ is the distance between the base points $(l)$ and the initial coordinates. We can demonstrate that the $K_{l}$ component is the relative distance between every point and the drawn plan (see formula below).

$$
M_{l, i}^{(2)}=M_{l, i}^{(1)}-K_{l}^{(1)} \cdot M_{c, i}^{(1)}
$$

After calculation the $K_{l}$ coefficient, we apply the previous formula only once to calculate the matrix lines, which form the projected points of the initial matrix. The Gram-Schmidt orthogonalization algorithm agrees on the constructed plan.

7. The second component $\left(R_{l}\right)$ that will be calculated is the distances on the plan, i.e., between the points projected on the plan and the point of the initial coordinates.

$$
R_{l}=\sqrt{\sum_{\mathrm{i}=1}^{\mathrm{m}}\left(M_{l, i}^{(2)}\right)^{2}}
$$

Finally, we obtain the $(K, R)$ components for each row of the original matrix $M^{1}$, as shown below.

$$
M^{1}=\left\|\begin{array}{llll}
x_{11} & x_{12} & \ldots & x_{1 m} \\
x_{21} & x_{22} & \ldots & x_{2 m} \\
x_{l 1} & x_{l 2} & \ldots & x_{l m} \\
x_{N 1} & x_{N 2} & \ldots & x_{N m}
\end{array}\right\|\left\|\begin{array}{cc}
K_{1} & R_{1} \\
K_{2} & R_{2} \\
K_{l} & R_{l} \\
K_{N} & R_{N}
\end{array}\right\|
$$

Thus, the new $(K, R)$ components generated by the GramSchmidt algorithm are ready to pass in the training phase 
After that, the MCP-ANNs outputs should be analyzed to show the performance of the combination between GSHM and CP-ANNs and compare them with the original network abilities.

\section{RESUlts}

\section{A. Performance of the CP-ANNs Model}

In the training phase of the CP-ANNs, each dataset was run with different training parameter values (see Table IV). The performance of the CP-ANNs during the learning process in all datasets is described below.

\section{1) The Correlation Dataset}

The experimental results show that the performance of the $\mathrm{CP}-\mathrm{ANNs}$ in the Correlation dataset is unqualified enough and unsatisfying due to the inability of the CP-ANNs to reach some desired outputs (see Table V). This limitation comes from the low level of the pattern recognition of the KohonenSelf-organizing map, which reduces the number of correct classifications. Here, the K-SOM gives five winning nodes for the eight input vectors (i.e., the K-SOM classifies the data into five independent clusters), as shown in Fig.5. The cause of decreasing the classification performance of the K-SOM depends on some input vectors having a linear regularities problem between their components (i.e., inputs $0,4,2$, and 6), that has an effect on the network performance by giving a single winning node for each pair instead of four, as shown in (Fig. 6 and 7). In this case, the Grossberg layer fails to provide good results. Thus, the $\mathrm{CP}-\mathrm{ANNs}$ are unable to reach all desired outputs (see Table V). For similar objects (i.e., inputs 1 and 5), the network offers the same winning node, as shown in Fig.9.

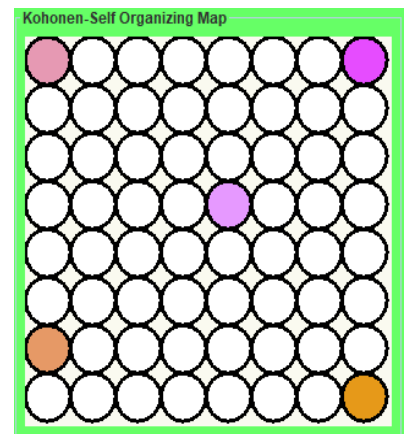

Fig. 5. The capability of the K-SOM in the classification performance of the Correlation Dataset, according to the CP-ANNs.

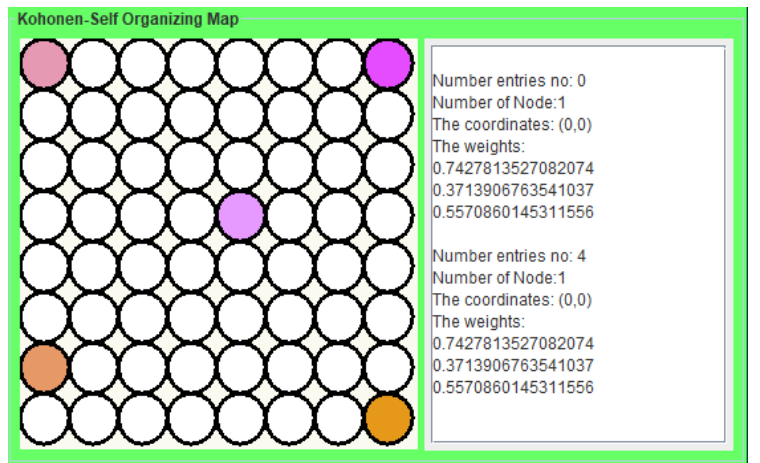

Fig. 6. The winning node of inputs 0 and 4 .

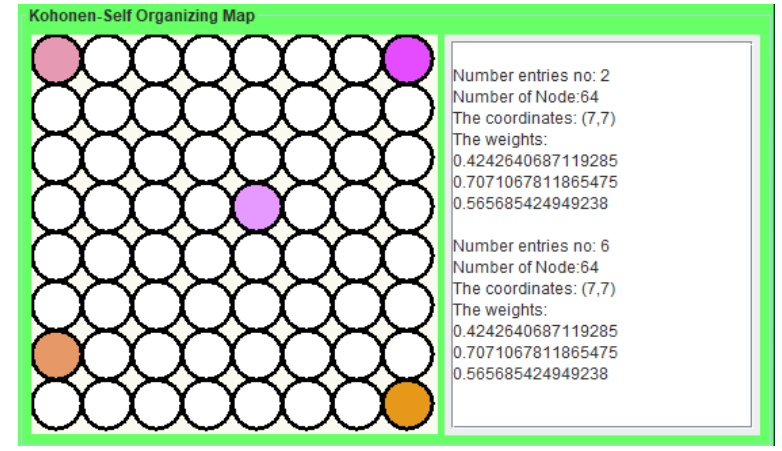

Fig. 7. The winning node of inputs 2 and 6.

From Fig. 6 and 7, the inputs 0 and 4 have the same winning node. In addition, inputs 2 and 6 also get the same winning node. Therefore the network produces two winning nodes from four input vectors instead of four. The coordinates of each winning node are described in Fig.8.

In Table $\mathrm{V}$, the $\mathrm{K}$-SOM classification represents the coordinates (or position $(\mathrm{y}, \mathrm{x})$ ) of the winning nodes.

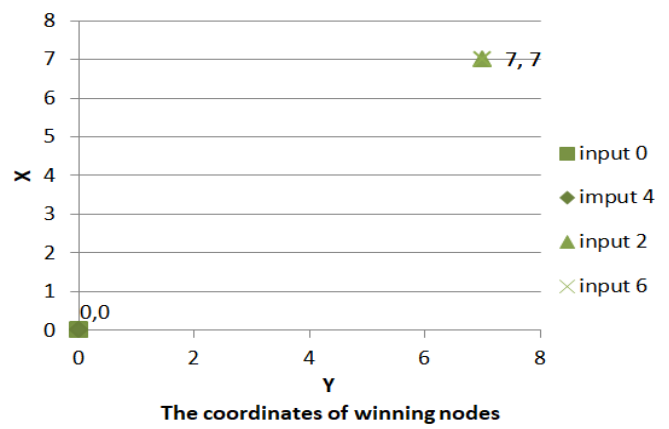

Fig. 8. The performance of the K-SOM in four input vectors that have a linear regularity between their components, according to the $\mathrm{CP}-\mathrm{ANNs}$.

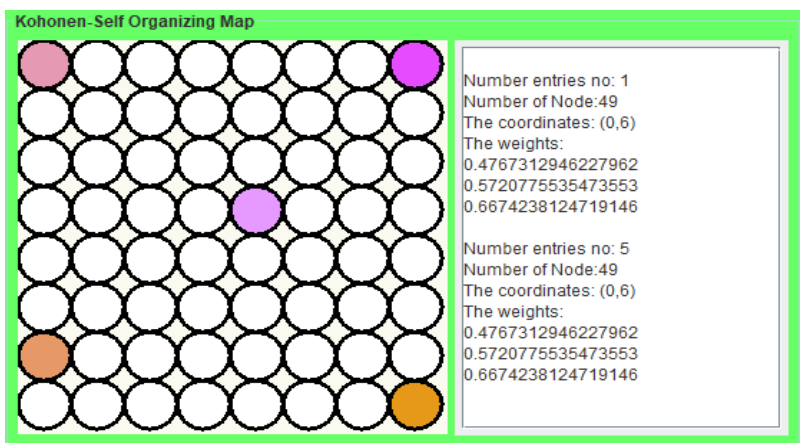

Fig. 9. The winning node of the similar input vectors 1 and 5 .

Accordingly to the results obtained, the CP-ANNs achieve a classification accuracy of $62.5 \%$ (i.e., 5/8). For training parameter values, the learning process results in the Correlation dataset provide (Max iteration $=523$, the mean error of $\mathrm{K}-\mathrm{SOM}=4.4018 \mathrm{e}^{-4}$, and mean error of Grossberg = $2.2684 \mathrm{e}^{-4}$ ) (see Table IV).

\section{2) The Crop and the Fertilizer Datasets}

The experimental tests of the CP-ANNs in the Crop and the Fertilizer datasets show high classification performance due to reaching all desired outputs with an accuracy of $100 \%$ (See Table V), but with these training parameter values (i.e., Max iteration $=779$, Mean error of the $\mathrm{K}-\mathrm{SOM}=6.1990 \mathrm{e}^{-4}$, and 
mean error of the Grossberg $=8.9907 \mathrm{e}^{-4}$ ) for the Crop dataset, and (Max iteration $=3000$, mean error of the K-SOM $=1.5712 \mathrm{e}^{-4}$, and mean error of the Grossberg $\left.=7.9210 \mathrm{e}^{-6}\right)$ for the Fertilizer dataset (see Table IV).

TABLE IV

The TRaining Parameter VAlues OF THE CP-ANNs Using THREE EXAMPLES OF DATASETS

\begin{tabular}{|c|c|c|c|}
\hline \multirow{2}{*}{$\begin{array}{l}\text { Training } \\
\text { parameters }\end{array}$} & \multicolumn{3}{|c|}{ Datasets Name } \\
\hline & Correlation & Crop & Fertilizer \\
\hline $\begin{array}{c}\text { Map } \\
\text { Dimension }\end{array}$ & 8 & 8 & 15 \\
\hline $\begin{array}{c}\text { Iteration } \\
\text { rate }\end{array}$ & 1000 & 1000 & 3000 \\
\hline $\begin{array}{l}\text { Max. } \\
\text { iteration }\end{array}$ & 523 & 779 & 3000 \\
\hline $\begin{array}{c}\text { Learning } \\
\text { error }\end{array}$ & 0.001 & 0.001 & 0.00001 \\
\hline $\begin{array}{l}\text { Mean error } \\
\text { of K-SOM }\end{array}$ & $4.4018 \mathrm{e}^{-4}$ & $6.1990 \mathrm{e}^{-4}$ & $1.5712 \mathrm{e}^{-4}$ \\
\hline $\begin{array}{c}\text { Mean error } \\
\text { of } \\
\text { Grossberg }\end{array}$ & $2.2684 \mathrm{e}^{-4}$ & $8.9907 \mathrm{e}^{-4}$ & $7.9210 \mathrm{e}^{-6}$ \\
\hline
\end{tabular}

TABLE V

THE EXPERIMENTAL PERFORMANCE OF THE CP-ANNS Using THREE EXAMPLES OF DATASETS

\begin{tabular}{|c|c|c|c|c|c|}
\hline \multicolumn{6}{|c|}{ Correlation Dataset (Example 1) } \\
\hline \multicolumn{2}{|c|}{$\begin{array}{l}\text { Input } \\
\text { vector } \\
\mathrm{N}^{\circ}\end{array}$} & $\begin{array}{l}\text { Desired } \\
\text { Output }\end{array}$ & CP-ANNs Output & $\begin{array}{l}\text { K-SOM } \\
\text { Classification }\end{array}$ & $\begin{array}{l}\text { Execution } \\
\text { Time }\end{array}$ \\
\hline \multirow{8}{*}{$\begin{array}{l}\frac{0}{2} \\
\frac{0}{\pi} \\
\frac{\pi}{4} \\
\mathbb{8}\end{array}$} & 0 & 1 & 5.000241999012946 & {$[0,0]$} & \multirow{8}{*}{$119 \mathrm{~ms}$} \\
\hline & 1 & 2 & 1.9999756799349575 & {$[0,6]$} & \\
\hline & 2 & 3 & 6.00013863576897 & {$[7,7]$} & \\
\hline & 3 & 4 & 4.000265373781499 & {$[4,3]$} & \\
\hline & 4 & 5 & 5.000241999012946 & {$[0,0]$} & \\
\hline & 5 & 2 & 1.9999756799349575 & {$[0,6]$} & \\
\hline & 6 & 6 & 6.00013863576897 & {$[7,7]$} & \\
\hline & 7 & 7 & 7.00022684372184 & {$[7,0]$} & \\
\hline \multicolumn{6}{|c|}{ Crop Dataset (Example 2) } \\
\hline & 0 & 5 & 4.999534540663555 & {$[7,2]$} & \multirow{9}{*}{$116 \mathrm{~ms}$} \\
\hline \multirow{8}{*}{$\frac{2}{2}$} & 1 & 20 & 19.999712136522206 & {$[3,5]$} & \\
\hline & 2 & 1 & 0.9995321693464999 & {$[6,4]$} & \\
\hline & 3 & 87 & 86.99978495693603 & {$[4,1]$} & \\
\hline & 4 & 123 & 122.99959283025792 & {$[0,3]$} & \\
\hline & 5 & 1234 & 1233.999291569404 & {$[7,7]$} & \\
\hline & 6 & 67 & 66.9996038867822 & {$[0,7]$} & \\
\hline & 7 & 250 & 249.99972761287634 & {$[6,0]$} & \\
\hline & 8 & 90 & 89.99910092045408 & {$[1,0]$} & \\
\hline \multicolumn{6}{|c|}{ Fertilizer Dataset (Example 3) } \\
\hline \multirow{9}{*}{\begin{tabular}{l} 
目 \\
$\frac{0}{2}$ \\
\multirow{\pi}{\pi}{} \\
$\stackrel{8}{0}$ \\
0
\end{tabular}} & 0 & 1 & 0.9999946186691387 & {$[0,10]$} & \multirow{9}{*}{$232 \mathrm{~ms}$} \\
\hline & 1 & 2 & 1.9999922567653823 & {$[14,14]$} & \\
\hline & 2 & 1 & 0.9999961747772984 & {$[3,14]$} & \\
\hline & 3 & 1 & 0.9999942897648376 & {$[2,11]$} & \\
\hline & 4 & 2 & 1.999996454285112 & {$[11,0]$} & \\
\hline & 5 & 1 & 0.9999947559140254 & {$[0,14]$} & \\
\hline & 6 & 2 & 1.9999931506204915 & {$[8,0]$} & \\
\hline & I & & & & \\
\hline & 37 & 2 & 1.999992078912501 & {$[13,9]$} & \\
\hline
\end{tabular}

In Conclusion, the performance of the CP-ANNs in the Crop and the Fertilizer datasets was high. In addition, the $\mathrm{CP}$ ANNs get an accuracy of $100 \%$ in the training, test, and validation sets. However, the CP-ANNs in the Correlation dataset demonstrate the need to add another technique to improve it due to very low classification accuracy $(62.5 \%)$. For that, our objective is to develop a new approach based on a combination of the Modified Gram-Schmidt algorithm and the CP-ANNs to improve classification accuracy by eliminating the ambiguities detected from the Correlation dataset and reducing the training parameter values and execution time in all datasets used.

\section{B. Performance of the Proposed MCP-ANNs approach}

In this test, the modified GSHM affords $(\mathrm{K}, \mathrm{R})$ components of each row of a given $\mathrm{r} x \mathrm{c}$ matrix (see Table VI). Hence, these components were transmitted to the training phase.

The proposed MCP-ANNs were trained with the same training parameter values as the CP-ANNs (see Table VIII).

\section{1) The Correlation Dataset}

It can be noticed from Table VII that the proposed MCPANNs improve the classification accuracy of the classical network by reaching all desired outputs. This improvement is back from the good pattern recognition in the K-SOM layer, which accurately classified the input-vectors as shown in Fig. 10 versus five different clusters for the CP-ANNs, as shown in Fig. 5. In the K-SOM classification, the inputs that have a linear regularities problem between their components (inputs $0,4,2$, and 6 ), the network gives a different winning node for each input, as shown in Fig. 11 compared to the CP-ANNs (see Fig. 8).

Thus, the K-SOM correctly classifies all input vectors, which gives the ability for the Grossberg layer to reach good results. These outcomes allow increasing the network classification performance by the accuracy of $100 \%$ and with a small mean error of the $\mathrm{K}-\mathrm{SOM}=3.5632 e^{-4}$ versus 4.4018 $e^{-4}$ for the CP-ANNs (see Table VIII).

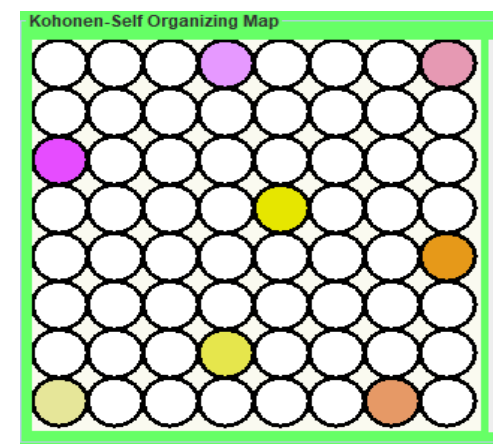

Fig. 10. The capability of the K-SOM in the classification performance of the Correlation Dataset, according to the MCP-ANNs.

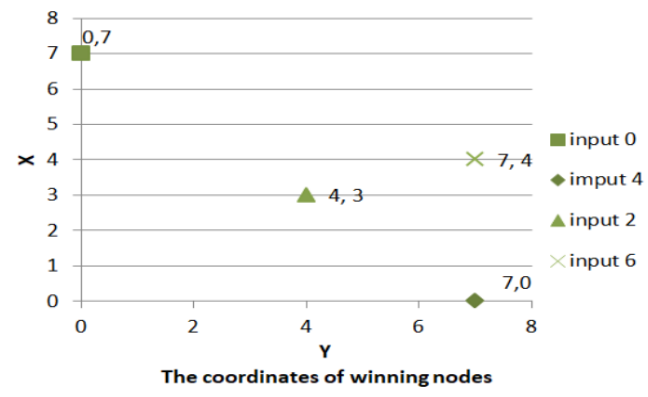

Fig. 11. The performance of the K-SOM in four input vectors that have a linear regularity between their components, according to the MCP-ANNs. 


\section{2) The Crop and the Fertilizer Datasets}

Moreover, the MCP-ANNs are designed to not only aim to solve the classification problem but also to reduce the training parameter values during the learning process, such as max iteration and mean error. In this experimental test, we can observe a significant minimization of the training parameter values of the Crop dataset in terms of $\max$ iteration $=656$, mean error of the K-SOM=3.0540 $e^{-4}$, and mean error of the Grossberg $=3.2211 e^{-5}$ compared to a $\max$ iteration $=779$, mean error of the $\mathrm{K}-\mathrm{SOM}=6.1990 e^{-4}$, and mean error of the Grossberg= $8.9907 \mathrm{e}^{-4}$ for the CP-ANNs (see Table VIII). The empirical test of the Fertilizer dataset gave the small mean error of the Grossberg $=4.5406 e^{-6}$ versus $7.9210 e^{-6}$ for the CP-ANNs (see Table VIII). Besides that, we obtained a similar classification accuracy of $100 \%$ for both of the CPANNs and the MCP-ANNs in training, test, and validation sets. The desired outputs and the MCP-ANNs output obtained for the Crop and the Fertilizer datasets during the learning process are described in Table VII.

In addition, the proposed approach reduces the execution time in all datasets during the learning process.

TABLE VI

THE K, R COMPONENTS GENERATING BY THE GSHM ALGORITHM OF EACH DATASET

\begin{tabular}{|c|c|c|c|}
\hline \multirow{2}{*}{\multicolumn{2}{|c|}{$\begin{array}{l}\text { Input } \\
\text { Vector } \mathrm{N}^{\mathrm{o}}\end{array}$}} & \multicolumn{2}{|c|}{ New Learning Data Generating by the GSHM Algorithm } \\
\hline & & $\mathrm{K}$ & \multirow{2}{*}{$\begin{array}{l}\mathrm{R} \\
10.7703296 \\
\end{array}$} \\
\hline \multirow{8}{*}{ 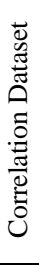 } & 0 & 0.0 & \\
\hline & 1 & 0.9137931 & 3.6246283 \\
\hline & 2 & 1.2277163 & 1.0758625 \\
\hline & 3 & 14.5674661 & $7.0340142 \mathrm{e}^{-14}$ \\
\hline & 4 & 1.8339636 & 1.5152288 \\
\hline & 5 & 5.2368931 & 6.8690373 \\
\hline & 6 & 11.3056469 & 11.8187847 \\
\hline & 7 & 31.5072648 & 90.9137290 \\
\hline \multirow{9}{*}{ 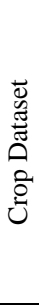 } & 0 & 0.0 & 1207.0879214 \\
\hline & 1 & 0.6385109 & 153.7390552 \\
\hline & 2 & 2.1390526 & 25.3022920 \\
\hline & 3 & 1.8806842 & 13.1382881 \\
\hline & 4 & 3.8361564 & 56.0178076 \\
\hline & 5 & 3.0579054 & 45.4536858 \\
\hline & 6 & 0.8321133 & 1.4887561 \\
\hline & 7 & 17.1582981 & $9.7498510 \mathrm{e}^{-11}$ \\
\hline & 8 & 15.8635010 & 3.9076074 \\
\hline \multirow{9}{*}{ 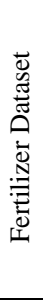 } & 0 & 0.0 & 78.7083223 \\
\hline & 1 & 0.9065375 & 43.8963008 \\
\hline & 2 & 2.2354967 & 6.8837815 \\
\hline & 3 & 3.7382374 & 2.4052783 \\
\hline & 4 & 3.1781479 & 7.7120735 \\
\hline & 5 & 5.3368075 & 8.2539199 \\
\hline & 6 & 4.8687976 & 1.1823540 \\
\hline & & & \\
\hline & 37 & 29.4138328 & 120.3985919 \\
\hline
\end{tabular}

\section{Comparison between the MCP-ANNs and the CP-ANNs}

\section{1) Classification Accuracy}

Figure 12 shows a comparison of classification accuracy between the MCP-ANNs and the CP-ANNs in all datasets. It can be observed from Fig 12 that the proposed MCP-ANNs improve the classification performance of the Correlation dataset with an accuracy of $100 \%$ against $62.5 \%$ for the CPANNs. For this dataset, the best improvement was $37,5 \%$ on average. This percentage represents an excellent improvement due to the ability of the proposed MCP-ANNs in removing all drawbacks or ambiguities detected from the Correlation dataset.

For the Crop and the Fertilizer datasets, we obtained the same classification performance with an accuracy of $100 \%$ of both the MCP-ANNs and the CP-ANNs, as shown in Fig.12.

TABLE VII

THE EXPERIMENTAL PERFORMANCE OF THE MCP-ANNS USING THREE EXAMPLES OF DATASETS

\begin{tabular}{|c|c|c|c|c|c|}
\hline \multicolumn{6}{|c|}{ Correlation Dataset (Example 1) } \\
\hline \multicolumn{2}{|c|}{$\begin{array}{l}\text { Input } \\
\text { Vector } \\
\mathrm{N}^{\mathrm{o}}\end{array}$} & $\begin{array}{l}\text { Desired } \\
\text { Output }\end{array}$ & MCP-ANNs Output & $\begin{array}{l}\text { K-SOM } \\
\text { Classification }\end{array}$ & $\begin{array}{l}\text { Execution } \\
\text { Time }\end{array}$ \\
\hline \multirow{8}{*}{ 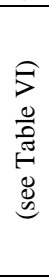 } & 0 & 1 & 1.0009755361207848 & {$[0,7]$} & \multirow{8}{*}{$90 \mathrm{~ms}$} \\
\hline & 1 & 2 & 1.9999355930620624 & {$[3,6]$} & \\
\hline & 2 & 3 & 2.9999681842404002 & {$[4,3]$} & \\
\hline & 3 & 4 & 3.9999919233796537 & {$[3,0]$} & \\
\hline & 4 & 5 & 4.999662299222258 & {$[7,0]$} & \\
\hline & 5 & 2 & 1.9999949926959124 & {$[6,7]$} & \\
\hline & 6 & 6 & 5.999910838500265 & {$[7,4]$} & \\
\hline & 7 & 7 & 6.999754071323308 & {$[0,2]$} & \\
\hline \multicolumn{6}{|c|}{ Crop Dataset (Example 2) } \\
\hline \multirow{9}{*}{ 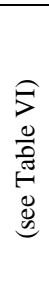 } & 0 & 5 & 5.000000317521804 & {$[7,0]$} & \multirow{9}{*}{$96 \mathrm{~ms}$} \\
\hline & 1 & 20 & 19.99994836365091 & {$[4,0]$} & \\
\hline & 2 & 1 & 0.9999099445880586 & {$[6,6]$} & \\
\hline & 3 & 87 & 86.99998573983048 & {$[3,6]$} & \\
\hline & 4 & 123 & 122.99991688651545 & {$[5,3]$} & \\
\hline & 5 & 1234 & 1233.9999692016274 & {$[7,3]$} & \\
\hline & 6 & 67 & 66.99970701428454 & {$[0,7]$} & \\
\hline & 7 & 250 & 249.99995884937874 & {$[0,0]$} & \\
\hline & 8 & 90 & 89.99996778890107 & {$[1,3]$} & \\
\hline \multicolumn{6}{|c|}{ Fertilizer Dataset (Example 3) } \\
\hline \multirow{9}{*}{ 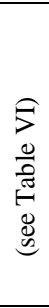 } & 0 & 1 & 0.9999927061766676 & {$[1,14]$} & \multirow{9}{*}{$173 \mathrm{~ms}$} \\
\hline & 1 & 2 & 1.9999903932358885 & {$[0,13]$} & \\
\hline & 2 & 1 & 0.9999932123688745 & {$[3,6]$} & \\
\hline & 3 & 1 & 0.9999930307466912 & {$[11,13]$} & \\
\hline & 4 & 2 & 1.9999959211943152 & {$[6,6]$} & \\
\hline & 5 & 1 & 0.9999958265352213 & {$[11,2]$} & \\
\hline & 6 & 2 & 1.999994646884562 & {$[6,11]$} & \\
\hline & $\ldots$ & $\ldots$ & 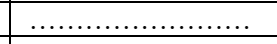 & ........ & \\
\hline & 37 & 2 & 1.9999954593314826 & {$[2,9]$} & \\
\hline
\end{tabular}

TABLE VIII

The Training PARAMETER VALUES OF THE MCP-ANNs USING THREE EXAMPLES OF DATASETS

\begin{tabular}{|c|c|c|c|}
\hline \multirow{2}{*}{$\begin{array}{l}\text { Training } \\
\text { parameters }\end{array}$} & \multicolumn{3}{|c|}{ Datasets Name } \\
\hline & Correlation & Crop & Fertilizer \\
\hline $\begin{array}{c}\text { Map } \\
\text { Dimension }\end{array}$ & 8 & 8 & 15 \\
\hline $\begin{array}{l}\text { Iteration } \\
\text { rate }\end{array}$ & 1000 & 1000 & 3000 \\
\hline $\begin{array}{c}\text { Max. } \\
\text { iteration }\end{array}$ & 638 & 656 & 3000 \\
\hline $\begin{array}{l}\text { Learning } \\
\text { error }\end{array}$ & 0.001 & 0.001 & 0.00001 \\
\hline $\begin{array}{l}\text { Mean error } \\
\text { of K-SOM }\end{array}$ & $3.5632 \mathrm{e}^{-4}$ & $3.0540 \mathrm{e}^{-4}$ & $2.0999 \mathrm{e}^{-4}$ \\
\hline $\begin{array}{c}\text { Mean error } \\
\text { of } \\
\text { Grossberg }\end{array}$ & $2.4592 \mathrm{e}^{-4}$ & $3.2211 \mathrm{e}^{-5}$ & $4.5406 \mathrm{e}^{-6}$ \\
\hline
\end{tabular}

\section{2) Mean Error}

As shown in Fig. 13 and 14, the proposed MCP-ANNs outperform the CP-ANNs in the mean error of the K-SOM and the Grossberg for the Crop dataset with the best improvement. Moreover, the MCP-ANNs minimize the max iteration 
number of this dataset against the CP-ANNs (see Table VIII and IV).

For the Correlation dataset, the MCP-ANNs produce a less mean error of the K-SOM versus the CP-ANNs as shown in Fig 13. Additionally, the MCP-ANNs show nearly the mean error of the Grossberg in comparison with the CP-ANNs, as shown in Fig. 14.

For the Fertilizer dataset, the MCP-ANNs give the best mean error of the Grossberg in comparison with the $\mathrm{CP}$ ANNs, as shown in Fig 14. Moreover, the MCP-ANNs generate a nearly similar mean error of the K-SOM as the CPANNs result (see Fig.13).

\section{3) Execution Time}

From the execution time point of view, Fig. 15 shows that the proposed MCP-ANNs also reduce the execution time in all training data (or datasets).

It can be concluded from these experimental tests that the proposed MCP-ANNs have a significant performance in terms of classification accuracy, training parameter values, and execution time.

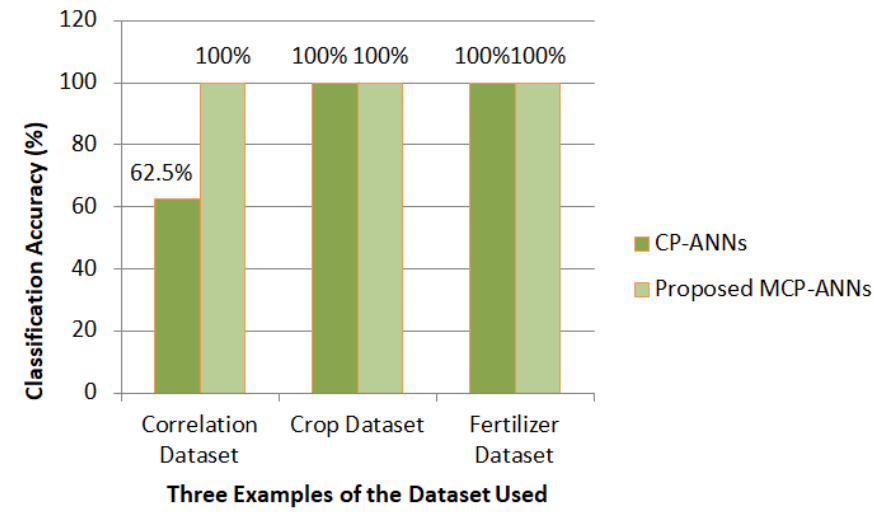

Fig. 12. The MCP-ANNs vs. the CP-ANNs in the classification accuracy.

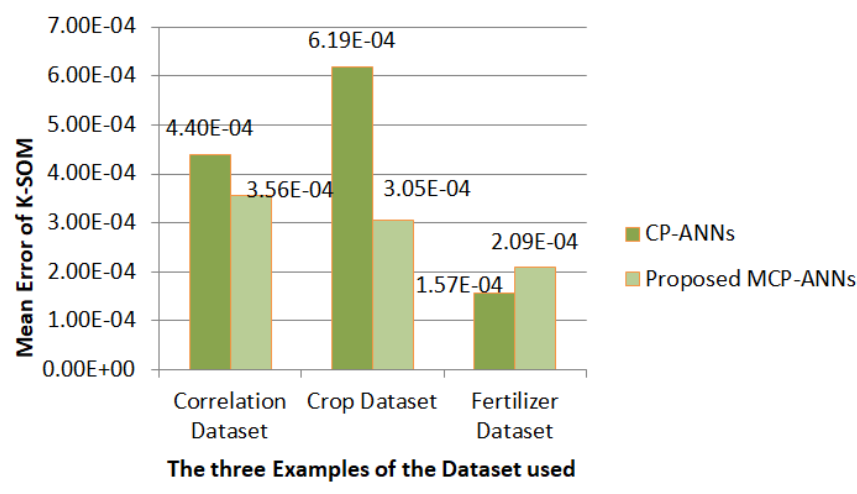

Fig. 13. The MCP-ANNs vs. the CP-ANNs in mean error of K-SOM.

\section{DISCUSSION}

Many different strategies have been proposed to improve the performance of the neural network algorithms, such as Kalman filter, particle swarm optimization (PSO), gravitational search algorithms (GSA), fuzzy rules, and recursive data pruning [13-15]. This study presents another new strategy called the Gram-Schmidt algorithm as a pre- processing step of the Counter Propagation Artificial Neural Networks. The CP-ANNs used the GSHM algorithm to eliminate all drawbacks during the learning process. This transformation leads to improving the network performance. Some previous works suggested other approaches to remove the ambiguities detected during the learning process, such as Principal Component Analysis [21]. The problem of the PCA is the execution time because it takes a long time for data preprocessing versus the GSHM algorithm. In addition, the GSHM algorithm produces more accurate values than PCA. Although the high capabilities of the proposed MCP-ANNs in classification accuracy, sometimes they can face some limitations that occur when the new inputs generated by GSHM are very close to each other. Therefore, in this situation, the proposed approach may fail to increase the number of correct classifications.

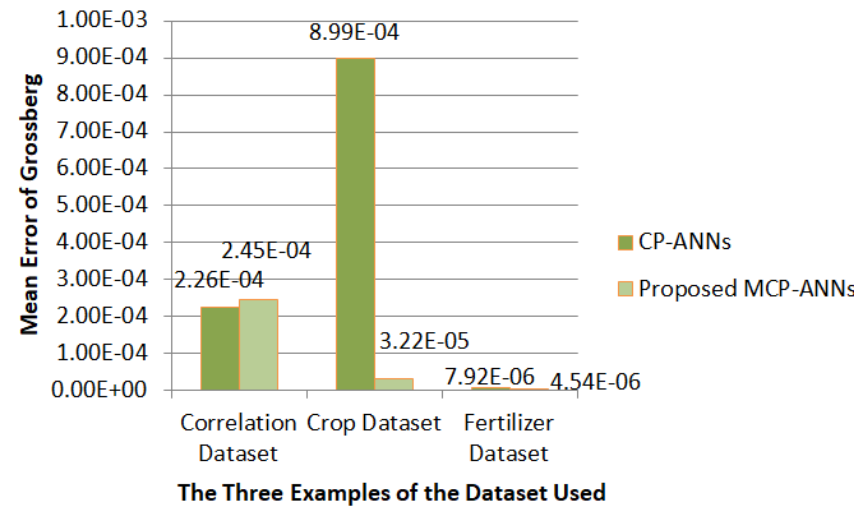

Fig. 14. The MCP-ANNs vs. the CP-ANNs in mean error of Grossberg.

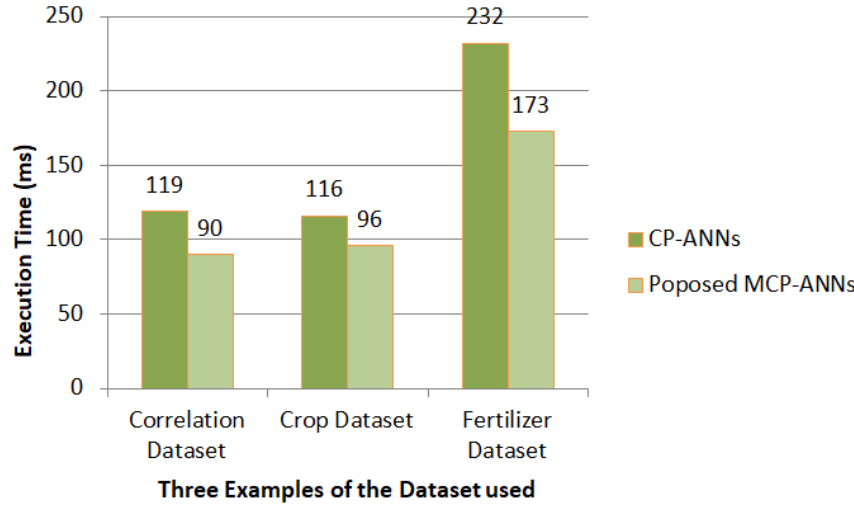

Fig. 15. The MCP-ANNs vs. the CP-ANNs in execution time.

\section{CONCLUSION}

This research presented a lower-cost and accurate Counter Propagation Artificial Neural Network based on the GramSchmidt algorithm. The purpose of improving the classical CP-ANNs is the ambiguities and drawbacks that they encountered during the learning process, which influences the first layer (K-SOM) by decreasing the pattern recognition and classification accuracies, resulting in unsatisfying outcomes in the second layer (Grossberg). Thus, this limitation reduces the general network performance. For that, the ultimate goal of the proposed approach is to provide a robust and efficient $\mathrm{CP}$ ANNs model which can be applied in different intelligent 
systems without worrying about some of its limitations. The proposed MCP-ANNs have been trained and tested using three datasets with different natures. The empirical results show that the proposed MCP-ANNs improve the classification accuracy $(100 \%)$ in the Correlation dataset due to good pattern recognition. For the Crop and the Fertilizer datasets, the proposed approach gives an accuracy of $100 \%$ in training, test, and validation sets. Furthermore, the proposed MCP-ANNs minimize some (or all) training parameter values and reduce the execution time in all datasets. In future research, we will try to test our proposed approach in various problems, especially in Hybrid and Diagnosis systems in order to show its efficacy.

\section{REFERENCES}

[1] F. Fernandes and G. Yen, "Pruning of generative adversarial neural networks for medical imaging diagnostics with evolution strategy". Information Sciences, 558, 91-102. https://doi.org/10.1016/ j.ins.2020. 12.086, (2021).

[2] I. Urbaniak and M. Wolter, "Quality assessment of compressed and resized medical images based on pattern recognition using a convolutional neural network". Communications in Nonlinear Science and Numerical Simulation, 95, 105582, https://doi.org/10.1016/j.cnsns. 2020.105582, (2021).

[3] K. Abdullah, B. Junaid, B. Javed Iqbal, K. Asfandyar, I. Muhammad, A. Muhammad, I. Muhammad, and K. Arshad, "Optimizing connection weights of functional link neural network using APSO algorithm for medical data classification". Journal of King Saud University- Computer and Information Sciences, xxxx. https://doi.org/10.1016/ j.jksuci.2020.10 $.018,(2020)$.

[4] L. Zhang, Z. Huang, W. Liu, Z. Guo, and Z. Zhang," Weather radar echo prediction method based on convolution neural network and Long ShortTerm memory networks for sustainable e-agriculture". Journal of Cleaner Production, 298, 126776.https://doi.org/10.1016/j.jclepro.2021 126776, (2021).

[5] G. Buyrukoğlu, S. Buyrukoğlu, and Z. Topalcengiz, "Comparing Regression Models with Count Data to Artificial Neural Network and Ensemble Models for Prediction of Generic Escherichia coli Population in Agricultural Ponds Based on Weather Station Measurements". Microbial Risk Analysis, December 2020, 100171. https://doi.org/10.101 6/j.mran.2021.100171, (2021).

[6] T. Khan, J. Qiu, M. Ali Qureshi, M. Iqbal, M. Rashid, and W. Hussain, “Agricultural Fruit Prediction Using Deep Neural Networks". Procedia Computer Science, 174(2019), 72-78. https://doi.org/10.1016/j.procs.20 20.06.058, (2020).

[7] Y. Jiang, C. Feng, B. He, J. Guo, D. Wang, and P. LV, “ Actuator fault diagnosis in autonomous underwater vehicle based on neural network". Sensors and Actuators, A: Physical, 324. https://doi.org/10.106/j.sna.20 21.112668, (2021).

[8] Z. Wang, W. Zhao, W. Du, N. Li, and J. Wang, "Data-driven fault diagnosis method based on the conversion of erosion operation signals into images and convolutional neural network". Process Safety and Environmental Protection, 149, 591-601. https://doi.org/10.1016/j.psep. 2021.03.016, (2021).

[9] J. Jiao, M. Zhao, J. Lin, and K. Liang, "A comprehensive review on convolutional neural network in machine fault diagnosis". Neurocomputing, 417, 36-63. https://doi.org/10.1016/j.neucom.2020. .07.088, (2020).

[10] Y. Li, Y. Pang, K. Wang, and X. Li, "Toward improving ECG biometric identification using cascaded convolutional neural networks". Neurocomputing, 391, 83-95. https://doi.org/10.1016/j.neucom.2020.0 1.019, (2020).

[11] S. Ashraf, Z. Aslam, S. Saleem, S. Afnan, and M. Aamer, "Multibiometric Sustainable Approach for Human Appellative". Transactions of Electrical, Electronic and Computer Engineering. Vol. 06(03), 146152, (2020).

[12] Yu. Chenglin and Pei. Hailong, "Face recognition framework based on effective computing and adversarial neural network and its implementation in machine vision for social robots". Computers and
Electrical Engineering, Volume 92, 2021, 107128, ISSN 0045-7906, https://doi.org/10.1016/j.compeleceng.2021.107128.

[13] M. Huang and Y. Chou, "Combining a gravitational search algorithm, particle swarm optimization, and fuzzy rules to improve the classification performance of a feed-forward neural network". Computer Methods and Programs in Biomedicine, 180. https://doi.org/10.1016/j. cmpb.2019.105016, (2019).

[14] Q. Li, P. Li, K. Mao, and E. Lo, "Improving convolutional neural network for text classification by recursive data pruning". Neurocomputing, 414, 143-152. https://doi.org/10.1016/j.neucom.2020 .07.049, (2020).

[15] J. Siswantoro, A. Prabuwono, A. Abdullah, and B. Idrus, "A linear model based on Kalman filter for improving neural network classification performance". Expert Systems with Applications, 49, 112122. https://doi.org/10.1016/j.eswa.2015.12.012, (2016).

[16] M. Mohammadi, M. Khorrami, H. Ghasemzadeh, P. Noor, and S. Zandbaaf, "Artificial neural network for quantitative and qualitative determination of the viscosity of nanofluids by ATR-FTIR spectrometry". Infrared Physics and Technology, 118(June), 103900. https://doi.org/10.1016/j.infrared.2021.103900, (2021).

[17] A. Mohammadian, M. Barzegar, and A. Mani-Varnosfaderani, "Detection of fraud in lime juice using pattern recognition techniques and FT-IR spectroscopy". Food Science and Nutrition, 9(6), 3026-3038. doi:10.1002/fsn3.2260, (2021).

[18] E. Farmaki, N. Thomaidis, I. Pasias, N. Rousis, C. Baulard, L. Papaharisis, and C. Efstathiou, "Advanced multivariate techniques for the classification and pollution of marine sediments due to aquaculture “. Science of the Total Environment, 763 doi:10.1016/j.sc itotenv.2020. 144617, (2021).

[19] H. Yan, P. Li, G. Zhou, Y. Wang, B. Bao, Q. Wu, and S. Huang, "Rapid and practical qualitative and quantitative evaluation of non-fumigated ginger and sulfur-fumigated ginger via fourier-transform infrared spectroscopy and chemometric methods". Food Chemistry, 341 doi:101 016/j.foodchem.2020.128241, (2021).

[20] S. Ashraf, S. Saleem, T. Ahmed, Z. Aslam, and D. Muhammad, “ Conversion of adverse data corpus to shrewd output using sampling metrics": Vis. Comput. Ind. Biomed. Art 3, 19. https://doi.org/10.1186// s42492-020-00055-9, (2020).

[21] H. El khatir , C. Loubna, F. Hanane, and E. Mostafa, "Towards a New Approach to Improve the Classification Accuracy of the Kohonen's SelfOrganizing Map During Learning Process”. (IJACSA) International Journal of Advanced Computer Science and Applications, Vol. 7, No. 3, (2016).

[22] O. I. Abiodun et al, "Comprehensive Review of Artificial Neural Network Applications to Pattern Recognition". IEEE Access, 7(February 2017), 158820-158846. https://doi.org/10.1109/ACCESS.2019.2945545, (2019).

[23] B. Sara, A. Otman, and H. El khatir, "New Learning Approach for Unsupervised Neural Networks Model with Application to Agriculture Field", International Journal of Advanced Computer Science and Applications (IJACSA), 11(5), 2020. http://dx.doi.org/10.14569/IJACSA .2020.0110548, (2020).

[24] P. Mohan and K. Kumari Patil, "Weather and Crop Prediction Using Modified Self Organizing Map for Mysore Region”, REVA University, Computer Engineering, India, December 23-2017, International Journal of Engineering and Intelligent Systems, Vol.11, No .2, (2018).

[25] S. Dahikar, V. Rode, and P. Deshmukh, "An Artificial Neural Network Approach for Agricultural Crop Yield Prediction Based on Various Parameters", International Journal of Advanced Research in Electronics and Communication Engineering (IJARECE) Volume 4, Issue 1, January, (2015).

[26] https://www.kaggle.com/gdabhishek/fertilizer-prediction.

[27] S. Ashraf, D. Muhammad, M. Shuaeeb, and Z. Aslam, "Development of Shrewd Cosmetology Model Through Fuzzy Logic". Journal of Research in Engineering and Applied Sciences, 5(3), 93-99. https://doi .org/10.46565/jreas.2020.v05i03.003, (2020).

[28] K. Sara and S. Insoo, "Application of complex systems topologies in artificial neural networks optimization": An overview, Expert Systems with Applications, Volume 180, 2021, 115073, ISSN 0957-4174, https://doi.org/10.1016/j.eswa.2021.115073, (2021).

[29] C. Edson and S. Christian, "A stochastic model of voice generation and the corresponding solution for the inverse problem using Artificial 
Neural Network for case with pathology in the vocal folds", Biomedical Signal Processing and Control, Volume 68, 2021, 102623, ISSN 17468094, https://doi.org/10.1016/j.bspc.2021.102623, (2021).

[30] P. Florent, D. Christophe, and Z. Tianyu, "A robust solution of a statistical inverse problem in multiscale computational mechanics using an artificial neural network", Computer Methods in Applied Mechanics and Engineering, Volume 373, 2021, 113540, ISSN 0045-7825, https://doi.org/10.1016/j.cma.2020.113540, (2021)

[31] N. Kumar and J. Ohri, "Novel m-PSO-SVM based interface controller design for haptic system". Journal of Communications Software and Systems, 16(2), 188-196. https://doi.org/10.24138/jcomss.v16i2.1002, (2020).

[32] A. Bashiti, M. Malkawi, M. Khasawneh, and O. Murad, "A novel neurofuzzy model to detect human emotions using different set of vital factors with performance index measure". Journal of Communications Software and Systems, 14(1), 121-129. https://doi.org/10.24138/jcomss.Kv14i1.3 92, (2018).

[33] J. Zupan, M. Novic, and J. Gasteiger, Chemometr. Intel. Lab. Syst. 27 175-187, (1995).

[34] R. Hecht-Nielsen, : Applications of counter propagation networks. Neural Networks. 1, 131-139 (1988).

[35] F. Marini, J. Zupan, and A. Magrì, Anal. Chim. Acta 510 231-240, (2004).

[36] W. Melssen, B. Ustun, and L. Buydens, Chemometr. Intel. Lab. Syst. 86 102-120, (2007)

[37] W. Melssen, R. Wehrens, and L. Buydens, Chemometr. Intel. Lab. Syst. 83 99-113, (2006).

[38] S. Leandro, A. Pimentel, F. Telmo, and S. Renata," Kohonen map-wise regression applied to interval data", Knowledge-Based Systems, Volume 224, 2021, 107091, ISSN 0950-7051, https://doi.org/10.1016/j.knosys.2021.107091, (2021).

[39] F. Troncoso, ClasSOMfier, " A neural network for cluster analysis and detection of lattice defects", Computational Materials Science,Volume 188, 2021, 110167, ISSN 0927-0256, https://doi.org/10.1016/j.commatsci.2020.110167, (2021).

[40] B. Ripley, Pattern recognition and neural networks. Cambridge: Cambridge University Press, NewYork (1996).

[41] S. Hosseini, "Binary Tree Time Adaptive Self-Organizing Map", Neurocomputing, Vol.74, No.11, pp.1823-1839, May 2011.

[42] M. Vracko, "Kohonen Artificial Neural Network and Counter Propagation Neural Network in Molecular Structure-Toxicity Studies". Current Computer Aided-Drug Design. 1, 73-78(2005).

[43] P. Ravisankar and V. Ravi, "Financial distress prediction in banks using Group Method of Data Handling neural network, counter propagation neural network and fuzzy ARTMAP”. Knowledge-Based Systems. 23, 823-831 (2010).

[44] L. Kohaupt, "Introduction to a Gram-Schmidt-type biorthogonalization method". Rocky Mountain Journal of Mathematics, 44(4), 1265-1279. https://doi.org/10.1216/RMJ-2014-44-4-1265, (2014).

[45] A. Bjorck, (1967). GRAM-SCHMIDT ORTHOGONALIZATION. 7.

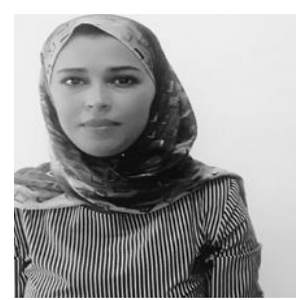

Sara Belattar received a Fundamental License degree in Computer Science and Mathematics from the Abdelmalek Essaadi University (UAE), Polydisciplinary Faculty, Morocco in 2016. Then, she obtained a Master's degree in Advanced Technologies and Embedded System at the same university in 2018. Currently, she is pursuing a $\mathrm{PhD}$ in Artificial Intelligence with Computer Science Department. Sara Belattar participated in several International Conferences and her research interest in Artificial Neural Networks.

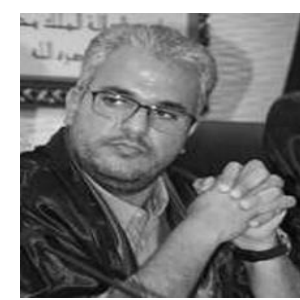

Otman Abdoun is a qualified professor in Computer Science at the Abdelmalek Essaadi University (UAE), Polydisciplinary Faculty, Morocco with Computer Science Departement. Currently, $\mathrm{He}$ is the coordinator of the IT Management Professional License (LPIG) at the same University. His research includes MetaHeuristics methods, Multi-Agents Systems, Artificial Intelligence, Computational intelligence, and more. Otman Abdoun has several publications and conference articles on previously cited topics.

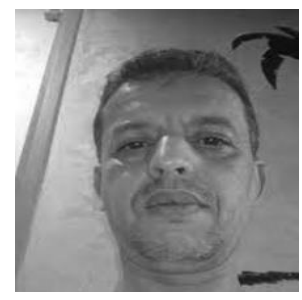

El Khatir Haimoudi received his Ph.D. in Artificial intelligence systems and resources especially in the recognition of multiparametric objects at the basis of artificial neural networks from the University of Ukraine. Currently, he is a qualified Professor at the Abdelmalek Essaadi University (UAE), Polydisciplinary Faculty, Morocco with Computer Science Departement. Furthermore, he is a coordinator of the Master "Intelligent Systems and Development of Decision-Making Systems" since 2019. His research includes Artificial Neural Networks, Classification, Clustering, Pattern Recognition, Soft Computing, and more. El khatir haimoudi has several journal publications and conference articles. 